East African Medical Journal Vol. 87 No. 2 February 2010

WANDERING SPLEEN: CASE REPORT

G. N. Mwango, MBChB, BSc(Hum Anat), MMed (Diag. Radiol), Lecturer and I. M. Muriithi, MBChB, MMed (Diag. Radio), Tutorial Fellow, Department of Diagnostic Imaging and Radiation Medicine, College of Health Sciences, University of Nairobi, P.O. Box 20723- 00202, Nairobi, Kenya

Request for reprints to: Dr. G. N. Mwango, P.O. Box 2607 - 00202, Nairobi, Kenya

\title{
WANDERING SPLEEN: CASE REPORT
}

\author{
G. N. MWANGO and I. M. MURIITHI
}

\begin{abstract}
SUMMARY
Wandering spleens are rare clinical entities found more commonly in women aged 20-40 years. We report one such case found in a 24-year-old nulliparous woman who presented with low abdominal pains of sudden onset and splenomegaly. An emergency abdominal CT scan showed an enlarged spleen located in the right lumbar region and extending into the pelvis. There was a long splenic pedicle containing tortuous vessels. A review of literature and the postulated aetiological factors and associations are discussed.
\end{abstract}

\section{INTRODUCTION}

Wandering spleen, also known as 'ectopic', 'proptopic', 'floating', 'displaced', or 'aberrant' spleen, is a rare clinical entity with less than 500 cases reported worldwide (1). It results from elongation or maldevelopment of the suspensory ligaments of the spleen (2). This condition occurs most commonly in women between 20 to 40 years of age $(3,4)$, the incidence of wandering spleen being 15 times higher in women in this age group than in men. It also occurs in children under the age of 10 years where the male to female ratio is 1:1 (3). While sporadic cases of wandering spleen have been reported worldwide even as early as 1667 by Hippocrates (2) and 11 cases seen in neighbouring Uganda (5), only one such case has been reported in Kenya (6).

\section{CASE REPORT}

A 24-year-old nulliparous woman presented at the emergency outpatient department of Kenyatta National Hospital (KNH) as a referral from a neighbouring private facility. The clinical records indicate that the patient presented with low abdominal pain of acute onset and a provisional diagnosis of splenomegaly. Abdominal sonograms done elsewhere gave conflicting diagnoses. The first scan reported a pelvic mass with the possibility of ectopic pregnancy while the second scan reported an enlarged pelvic spleen with a long pedicle of tortuous vessels. An emergency abdominal CT scan

done in the KNH outpatient department showed an enlarged spleen located in the right lumbar region and extending anterior and lateral to the uterus into the right adnexa (Figure $1 \mathrm{~A}-\mathrm{D})$. The uterus was non-gravid. The spleen showed normal perfusion with intravenous contrast medium (Figure $1 \mathrm{C}$ and D, Figure 2).

\section{Figure 1}

Axial post IV contrast CT scan images of the abdomen (Images $A$ to $C$ ) and pelvis(Image D) (arterial phase) showing a tortuous vascular pedicle containing the splenic vessels (white block arrow) and thickening of the splenic flexure and its mesentery from partial mesenteric ischemia (black arrow). A pelvic spleen (5) is demonstrated anterior to the uterus $(U)$

a

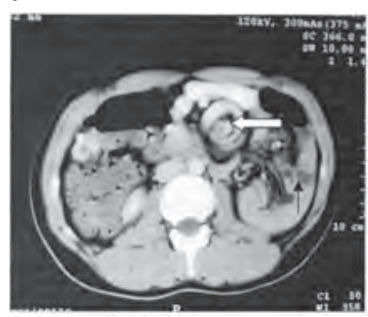

C

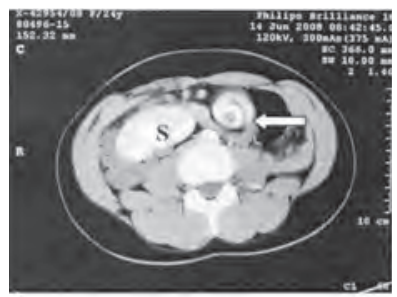

b

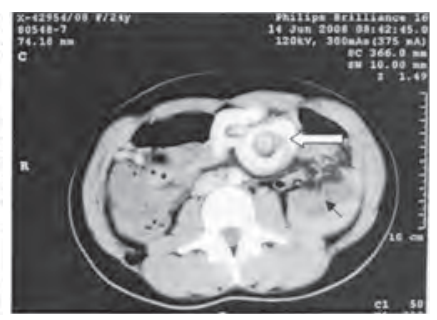

d

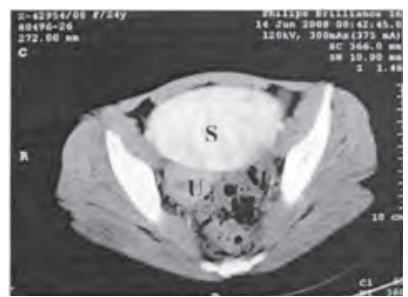




\section{Figure 2}

Coronal $(A)$ and sagittal $(B)$ reformatted $C T$ scan images of the abdomen post IV contrast administration (arterial phase) showing an enlarged spleen (5) in the right lumbar region extending anterior and lateral to the uterus $(U)$ into the right adnexa. The spleen shows normal perfusion. A long tortuous vascular pedicle containing the splenic vessels (white block arrow) is seen extending from the epigastric region to the prolapsed spleen.

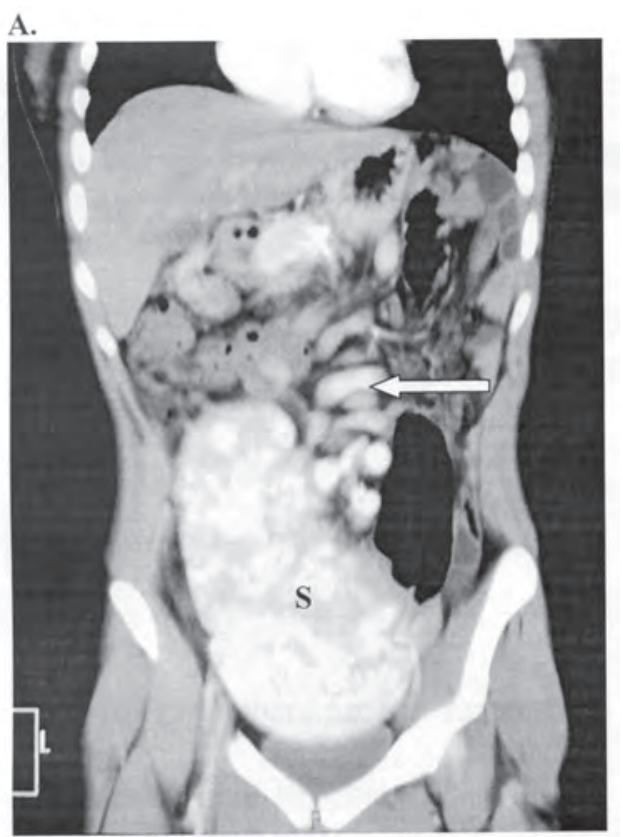

B.

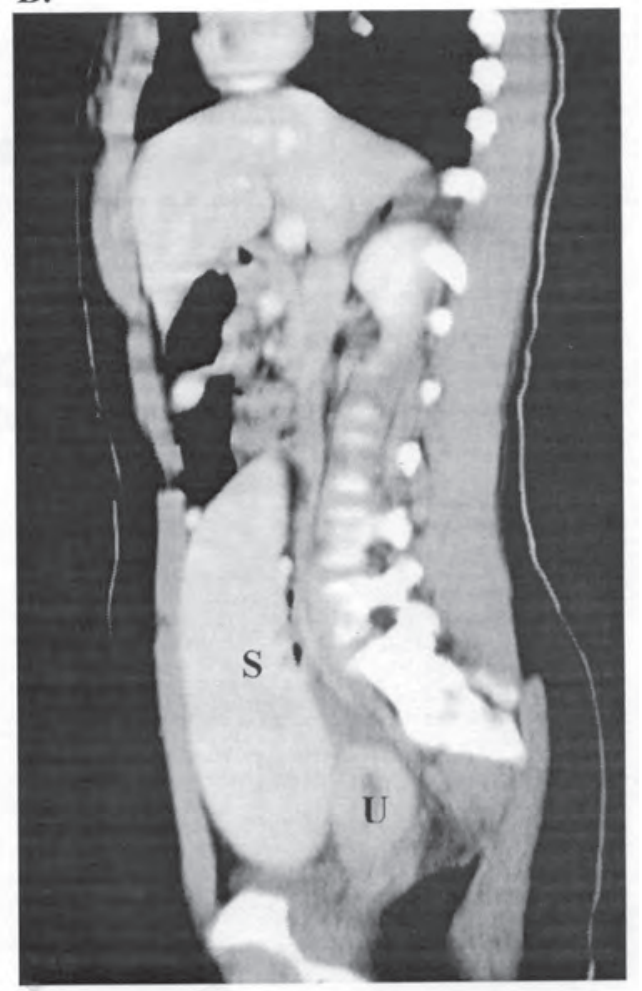

There was a long splenic pedicle containing tortuous vessels with the splenic vein encircling the artery (Figure 1A-C, Figure 2B), a shortened pancreatic tail and thickening of the splenic flexure and its mesentery from partial mesenteric ischemia (Figure $1 A$ and $B)$. The patient opted to continue with further management privately where open splenectomy was performed confirming the diagnosis. The patient did well on follow-up visits.

\section{DISCUSSION}

Wandering spleens are not considered in most textbooks as differential diagnoses for pelvic masses or acute abdomen due to their rarity. Embryologically, the spleen develops from mesenchymal clusters of cells within the dorsal mesogastrium during the 5 th week of intrauterine life (7). Its major supporting ligaments, which are derived from the dorsal mesogastrium, are the gastrosplenic, lienorenal, splenocolic and phrenicocolic ligaments (5). It is postulated that lack of fusion of the dorsal mesogastrium to the dorsal peritoneum results in the clinical entity of wandering spleen (2). The increased incidence in women has been attributed to an acquired ligamentous laxity caused by the hormonal effects of pregnancy $(2,4)$. A familial occurrence in two sisters has also been reported (9). An increased risk of wandering spleen has been reported in prune-belly syndrome, and in conditions that result in splenomegaly (2). Hypersplenism, thrombocytopenia and lymphoma have also been described in association with wandering spleen (10).

Whereas wandering spleens have been reported worldwide, there are fewer reported cases in the tropics where splenomegaly is a common condition. This seems to raise the question as to whether splenomegaly is a major aetiological factor. As the prolapsed spleen is usually enlarged, it is possible that this maybe the result of increased perfusion. The torqued splenic vessels can lead to splenic congestion with resultant splenomegaly (2). Thus, the splenic enlargement becomes a consequence of wandering spleen and not a predisposing factor. A reported case of spontaneous rupture of a wandering spleen (11) appears to support this theory where the increased intraparenchymal pressure from vascular congestion may have resulted in the ruptured spleen. Wandering spleen presents clinically with an abdominal mass or abdominal pain in $60 \%$ of cases, the rest being asymptomatic (3). Prompt diagnosis is necessary to prevent complications from torqued vessels such as splenic infarction. Other complications that can occur include gastric volvulus (12), portal hypertension 
with bleeding gastric varices (13), acute pancreatitis (14), intestinal obstruction( 6) and mesenteric and intestinal infarction, with signs of the latter seen in the CT scans of this patient (Figure $1 \mathrm{~A}$ and $\mathrm{B}$ ). Unusual presentations include in-utero torsion of wandering spleen (15) and torsion of wandering accessory spleens (16).

Diagnosis is usually established using imaging. Sonography is the preliminary method of evaluation for abdominal pain or masses but bowel gas may obscure findings (14). Color Doppler sonography will enable evaluation of the splenic vessels. However, if the spleen regains its normal or near normal position, the diagnosis maybe missed on sonography (14). Contrast enhanced abdominal CT scan is the investigation of choice and can be used to confirm the diagnosis and perfusion of the spleen following sonography, or as a preliminary imaging modality, in patients who present with acute abdomen. Abdominal CT can also delineate related complications or associations like pancreatic tail necrosis or diaphragmatic eventration (2). MRI has also been found to be a useful adjuvant imaging modality in the diagnosis of wandering spleen. It can provide information on the precise location of the spleen, the viability of the splenic parenchyma and the splenic vessel anatomy (17).

Management of the wandering spleen is surgical usually splenopexy (open or laparoscopic) in the uncomplicated spleen and splenectomy in cases of splenic infarction. Splenopexy has been reported to reverse the hypersplenism associated in the wandering spleen (10) and furthermore prevents post-splenectomy sepsis whose overall incidence is $2-4 \%$ with a mortality of $1-2 \%$ even with pneumococcal vaccination and daily antibiotic prophylaxis (2). Our case patient has been reported to be doing well to date.

\section{ACKNOWLEDGEMENTS}

To our colleagues Dr. B. Mugi and Dr. V. Manduku, Consultant Radiologists at Kenyatta National Hospital (KNH) and Kenya Medical Research Institute (KEMRI) respectively, for providing the clinical history and information on follow-up for the patient.

\section{REFERENCES}

1. Ugwu, A.C., Ogbonna, C.O. and Imo, A.O. Wandering spleen: A common presentation of an uncommon anomaly. Online. J. Health Sci. 2008; 7: 9.

2. Qazi, S.A., Mizra, S.M., Muhammad, A. et al. Wandering spleen (review article). Saudi. J. Gastroent. 2004; 10: 1-7.

3. Desai, D. C., Hebra, A., Davidoff, A. M. and Schnauffer, L. Wandering spleen: A challenging diagnosis. South Med. J. 1997; 90: 367- 462.

4. Zarrintan, S., Jamali, F., Tubbs, R.S., et al. A wandering spleen presenting as a pelvic mass: case report and review of literature. Folia Morphol. 2007; 66: 152-154.

5. Carswell, J.M. Wandering spleen: 11 cases from Uganda. Brit. J. Surg. 1974; 61: 495-497.

6. Githaiga, J.W. and Adwok, J.A. Wandering spleen presenting as a righthypochondrial mass and intestinal obstruction. East. Afr. Med. J. 2002; 79: 450-452.

7. Moore, K.L. The developing human: clinically oriented embryology. 2nd edition. Philadelphia: Saunders; 1977.

8. McMinn, R.M.H., editor. Last's anatomy: Regional and applied. 9th edition. Churchill Livingstone; 1994.

9. Elya, A.B., Segureb, E., Lotand, G., et al.. Familial wandering spleen: a first instance. J. Paed. Surg. 2008; 43: e23-e25.

10. Benoist, S., Imbaud, P. and Veyriere, M. Reversible hypersplenism after splenopexy of wandering spleen. Hepatogastroenterology. 1998; 45: 2430-2431.

11. Moran, J.C., Shah, U. and Singer, J.A. Spontaneous rupture of a wandering spleen: case report and literature review. Curr. Surg. 2003; 60: 310-312.

12. Spector, J.M. and Chapell, J. Gastric volvulus associated with wandering spleen in a child. J. Paed. Surg. 2000; 35: 641-642.

13. Daneshgar, S., Eras, P., Feldman, S., et al. Bleeding gastric varices and gastric torsion secondary to a wandering spleen. Gastroenterology. 1980; 79: 141-143.

14. Kamaryzyn, B., Steinberg, R., Gayer, G., et al. Wandering spleen; the challenge of ultrasound diagnosis: Report of 7 cases. J. Clin. Ultras. 2005; 33:433-438.

15. Nawaz, A., Jacobsz, A., Matta, H., et al. Intrauterine torsion of a wandering spleen presenting as an abdominal cystic swelling. J. Pediatr. Surg. 2000; 35: 1508-1510.

16. Valls, C., Mores, L., Guma, A. and Lopez-Calonge. Torsion of a wandering accessory spleen: CT findings. Abdom. Imag. 1998; 23: 194-195.

17. Deux, J. Acute torsion of wandering spleen: MRI findings. AJR. 2004; 182:1607- 1608. 